\title{
Biosynthesis of Silver Nanoparticles Using Annonamuricata Leaf Extract and its Antioxidant Activity
}

\author{
Parthiban, Rajeshwari, Jeyapratha, ChandraLekha
}

\begin{abstract}
Silver nanoparticles have been correctly synthesizedusingAnnonamuricata leaf extricate by the usage of eco-accommodating, minimum attempt technique. The nanoparticles have been defined through utilizing $U V$-vis, FTIR, EDAX,SEM and TEM one at a time. The UV/Vis variety of AgNPs confirmed a surface plasmon ingestion band with best absorbance at $429 \mathrm{~nm}$. FTIR spectra demonstrated the nearness of phytochemicals which might be in rate of the mixture of nanoparticles fromAnnonamuricata separate. Triangular states of severa sizes had been observed inside the geology of AFM photo of silver nanoparticles. SEM photographs of the silver nanoparticles regarded like agglomerated circles with ugly surfaces. TEM photo additionally verified the silvernano debris have been seen round match as a play around with a easy floor morphologyand the width of the nano debris became visible as spherical 50nm. The silver nanoparticles included from Annonamuricata leaf pay attention demonstrated the notable capability of maximum cancers prevention agent physical video games.
\end{abstract}

Keywords: Annonamuricata,silver nanoparticles, antioxidant activities.

\section{INTRODUCTION}

Nanoscience and innovation is one of the speedy growing fields because it has capacity packages inside the fields, as an example, optoelectronics[1],catalyst[2,3], gas-cellular[7,8,9] antibacterial[10,11], antimicrobial [12,13,14,15] and antifungal sports[16]. Nanoscience and nanotechnology especially manage the mixture, portrayal and research of numerous types of nanostructured materials.inexperienced combination of steel nanoparticles with healing plants is a trustworthy, relaxed and ecofriendly approach $[4,5,6]$ and has huge applications in restorative fields. plants are rather wealthy hereditary inconstancy and function numerous captivating phytochemicals that may reduce metal debris to nanoparticles in a solitary broaden. plants can along those lines fill in as proper away handy and biochemically rich hotspots for nanoparticle aggregate.

Revised Version Manuscript Received on 10 September, 2019.

Parthiban, Research scholar, Reg.No: 18212102031009, Department of Chemistry, Kamaraj College, Thoothukudi. (Affiliated to M.S University,Tirunelveli), Tamil Nadu, India.(email: chandralekha1978@yahoo.com)

Rajeshwari, PG student ,Department of Chemistry, Kamari College, Thoothukudi. (Affiliated to M.S University, Tirunelveli), Tamil Nadu, India.

Jeyapratha, Research scholar, Reg. No:19112102032001, Department of Chemistry, Kamaraj College, Thoothukudi. (Affiliated to M.S University,Tirunelveli), Tamil Nadu, India.

ChandraLekha, Department of Chemistry, Kamaraj College, Thoothukudi.(Affiliated to M.S University, Tirunelveli), Tamil Nadu, India.

\section{PLANT DESCRIPTION OF ANNONAMURICATA}

\subsection{Taxonomy}

\subsubsection{Common Names}

It is known by many common names such as; Soursop, Paw-Paw, Graviola,Guanabana, Adunu, Sorasaka, Mullaatha, Thorny custard apple, Shul-ram-fal, Hanuman fal. In Uganda, the Basoga call it Omusitafeli, while the Baganda call it Ekitafeli.

\subsubsection{Plant profile \\ Kingdom : Plantae \\ Division : Angiosperms \\ Class :Magnoliids \\ Order :Magnoliales \\ Family :Annonaceae \\ Genus :Annona \\ Species :Annonamuricata}

\section{III.METHODOLOGY:}

\subsection{Collection of Plant material and preparation of extract}

Annonamuricata leaves have been collected from Thoothukudi. They have been washed and wiped easy with sensitive water. Altogether washed leaves (a hundred g) were reduce and overflowed with a hundred $\mathrm{ml}$ of deionised water for $15 \mathrm{~min}$ in warming mantle at temperature eighty ${ }^{\circ} \mathrm{C}$. the subsequent object became separated and located away in refrigerator for in addition checks.

\subsection{Preparation of silver nitrate solution}

Seventeen milligrams (17 mg) of silver nitrate (Analytical evaluation) 99.nine\% AgNO3, MW = $169.87 \mathrm{~g} / \mathrm{mol}$ ) were gauged using electronic parity and moved into $500 \mathrm{ml}$ Erlenmeyer flagon. The silver nitrate grow to be step by step broken up through tenderly twirling the jar containing diffused deionized water. After all of the strong has disintegrated, greater water became step by step brought to carry the degree of arrangement precisely to a quantity feature of one hundred $\mathrm{ml}$. The readied $1 \mathrm{mM}$ silver nitrate arrangement become placed away at four ${ }^{\circ} \mathrm{C}$ in golden shaded jug.

\subsection{Synthesis of silver nanoparticles}

A amount of $20 \mathrm{ml}$ of watery listen of Annonamuricata

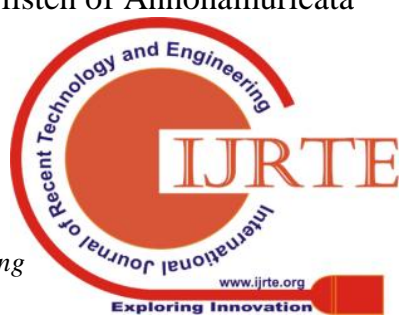


leaves changed into added to $80 \mathrm{ml}$ of one $\mathrm{mM}$ of silver nitrate affiliation in $100 \mathrm{ml}$ Erlenmeyer hued for decrease of Ag+ particles and adjustment of AgNPs.The responses had been finished in dimness (to maintain a strategic distance from image enactment of $\mathrm{AgNO} 3$ ) at room temperature. whole decrease of AgNO3to $\mathrm{Ag}+$ particles turned into affirmed with the aid of seen distinction in shading from slight green to ruddy darkish coloured confirmed the development of silver nanoparicles (AgNPs). The combination silver nanoparicles positioned away at $4{ }^{\circ} \mathrm{C}$ for in the future later.

\section{IV.RESULTS AND DISCUSSION}

\subsection{UV-Visible spectral study of AgNPs:}

it's miles extraordinary that AgNPs display a rosy darkish colored shading in watery affiliation because of excitation of floor plasmon vibrations. decrease of silver debris to AgNPs may be trailed with the useful resource of a shading exchange from pale dark colored to ruddy darker and which became stated by using visible research. The UV/Vis variety of AgNPs validated a ground plasmon assimilation band with maximum severe absorbance at $429 \mathrm{~nm}$ (Fig.1) displaying the nearness of everyday and all round scattered AgNPs. floorplasmon resonances are unmistakably blanketed within the optical spectra and had been placed in important locale. The most severe absorbance modified into visible at $429 \mathrm{~nm}$ which unmistakably demonstrates the age of AgNPs.

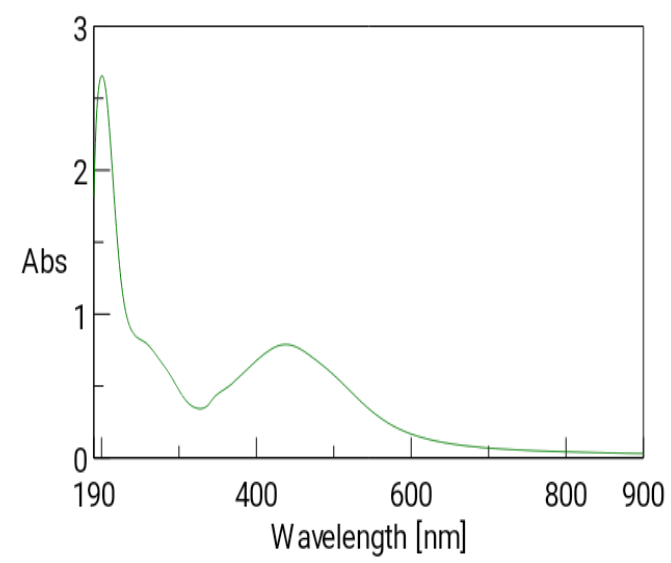

Figure : 1 UV-Vis spectrum of AgNPs

\subsection{FTIR spectroscopy:}

The FTIR range of powdered leaf changed into demonstrated in Fig.2. The IR range of powdered leaf of Annonamuricata demonstrated band at 3678.Eighty four $\mathrm{cm}-1,3408.93 \mathrm{~cm}-1$ relates to $\mathrm{O}-\mathrm{H}$ extending vibrations of alcohols and phenols , $2922.28 \mathrm{~cm}-1$ and $2852.50 \mathrm{~cm}-1$ compares to $\mathrm{C}-\mathrm{H}$ extending vibrations of alkenes and $1462.57 \mathrm{~cm}-1$ relates to $\mathrm{C}-\mathrm{H}$ twist extending vibrations of alkenes, 1409.Sixty cm-1 C-C extending aromatics. $1269.59 \mathrm{~cm}-1$ and $1155.67 \mathrm{~cm}-1$ relates to $-\mathrm{CH} 2 \mathrm{X}$ extending vibrations of alkyl halides.

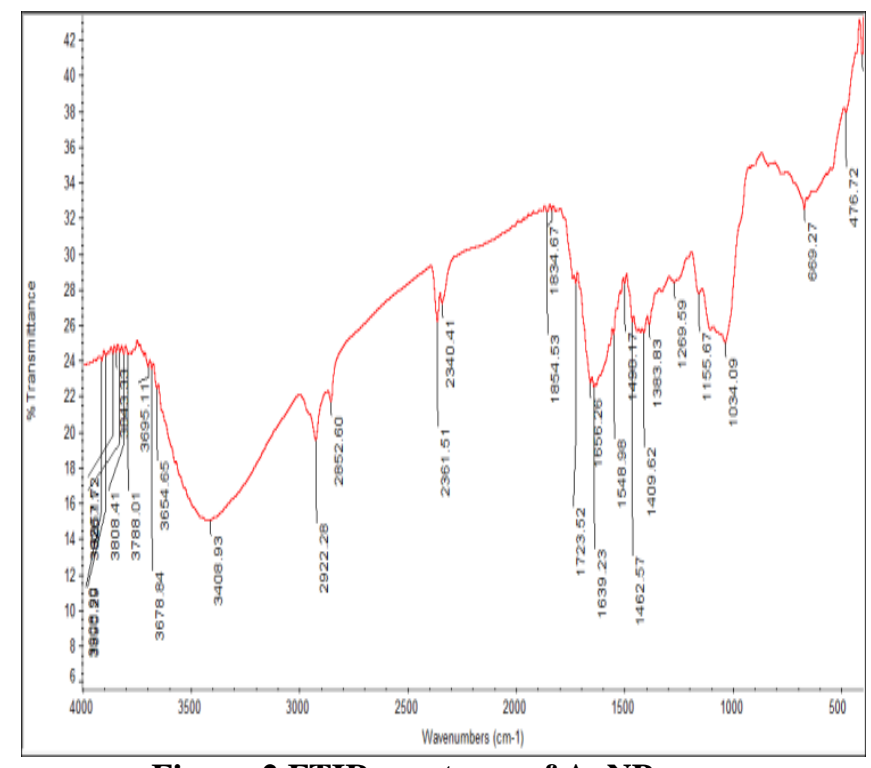

Figure:2 FTIR spectrum of AgNPs

\subsection{AFM studies}

AFM pictures were taken utilizing NanoSurfeasyscan 2 AFM(BT02218).Topography of the silver nanoparticles orchestrated from Annonamuricata concentrate were given in the Fig.3.Triangular states of various sizes were found in the geography. Some extended oval molded particles were available in the geology.

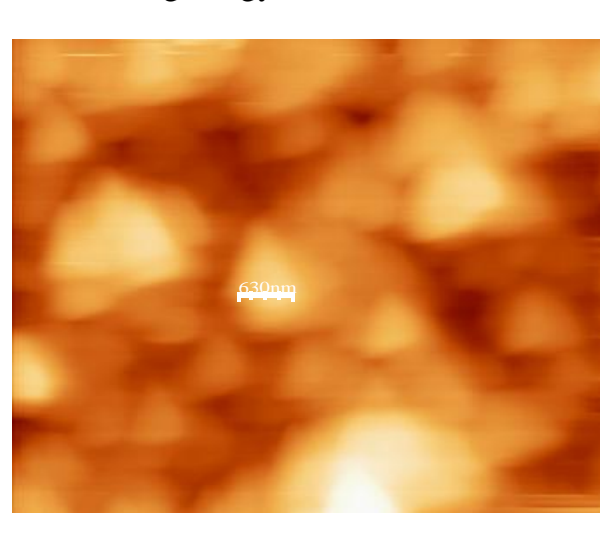

(a)

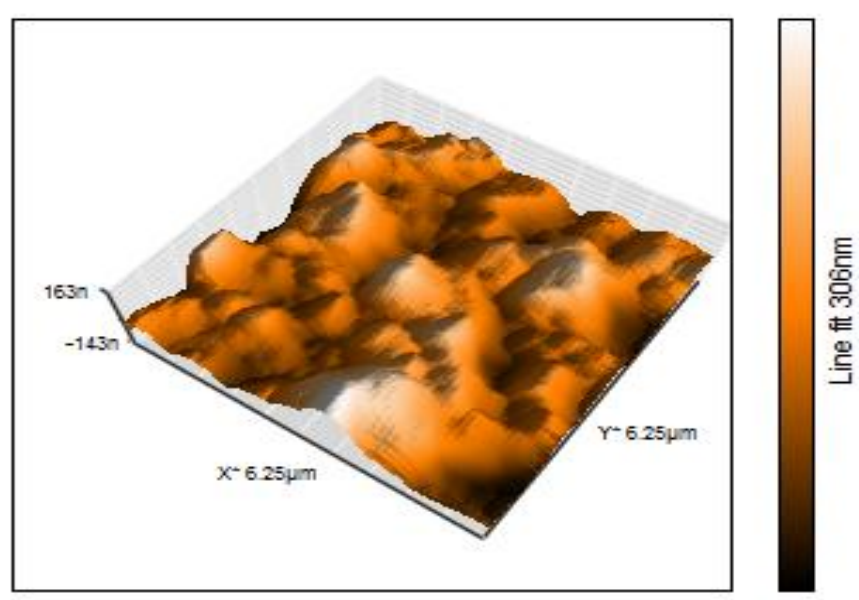

(b)

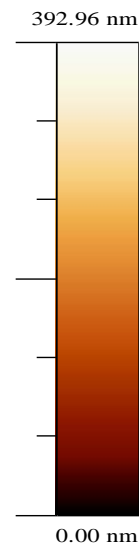

$0.00 \mathrm{~nm}$ 


\subsection{SEM-EDAX analysis of AgNPs:}

Scanning Electron Microscopy (SEM) was utilized to distinguish the morphology of the orchestrated silver nanoparticles as appeared in Figure 4. It was seen that states of the silver nanoparticles seemed like agglomerated circles with unpleasant surfaces. Additionally it was created the impression that these particles have harsh surfaces and might be made out of littler nanoparticles.

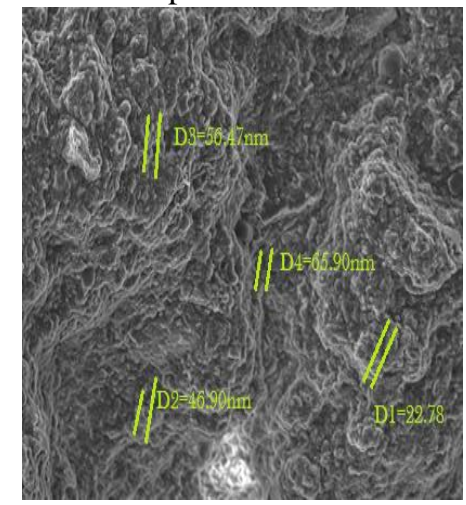

Figure :4 SEM image of AgNPs

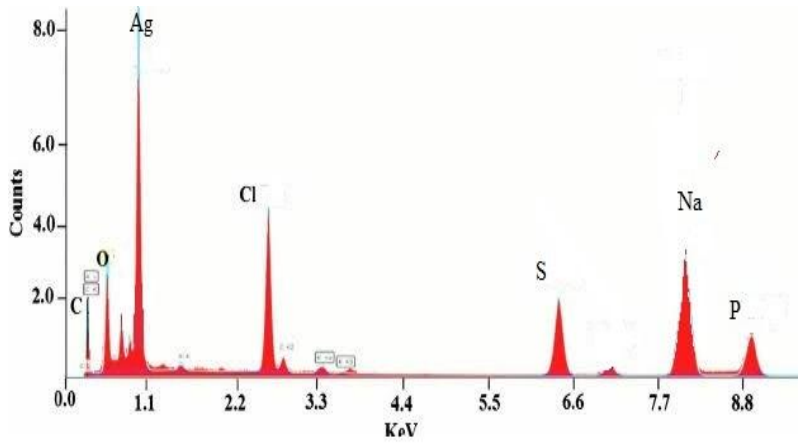

Figure :5 EDAX image of AgNPs

\begin{tabular}{|l|l|l|l|}
\hline Element & weight $\%$ & Atomic $\%$ & Net weight \\
\hline $\mathrm{Ag}$ & 72.24 & 77.92 & 432.09 \\
\hline $\mathrm{Cl}$ & 11.32 & 4.01 & 67.7 \\
\hline $\mathrm{Na}$ & 10.24 & 2.35 & 61.24 \\
\hline $\mathrm{O}$ & 2.19 & 0.35 & 13.09 \\
\hline $\mathrm{C}$ & 1.72 & 0.21 & 10.28 \\
\hline $\mathrm{S}$ & 1.25 & 0.4 & 7.65 \\
\hline $\mathrm{P}$ & 0.94 & 0.29 & 1.79 \\
\hline
\end{tabular}

\section{Table. 1 EDAX ofAgNPs}

4.5. High resolution transmission electron microscopy-HRTEM of AgNPS:

A TEM picture of the readied silver nano particles is appeared in the Fig.6. The Ag nano particles are round fit as a fiddle with a smooth surface morphology. The width of the nano particles is seen as roughly $50 \mathrm{~nm}$. TEM picture likewise demonstrates that the created nano particles are in shapes like triangle, bar and shell. The various shapes are because of the phytochemicals. Every phytochemical are in charge of the arrangement of specific shapes
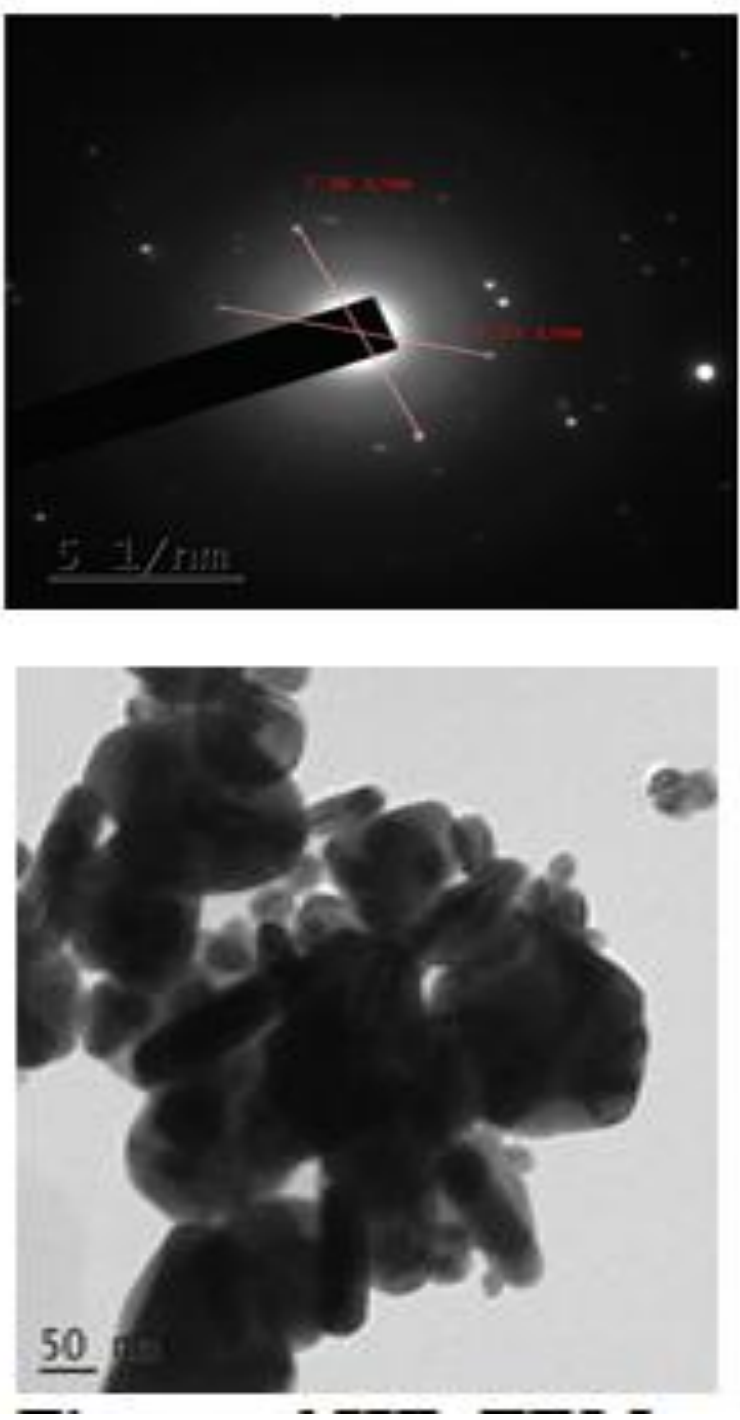

Figure :6 HR-TEM images of AgNPs

\subsection{Total Antioxidant Capacity:}

The total antioxidant capacity of the leaf extract was shown above Fig.7. The leaf extract possessed greater antioxidant potential. Total Antioxidant activity by Phosphomolybdenum method

$\%$ Antioxidant activity $=(1-($ Abs Std - Abs sample $/$ Abs Std) $) * 100$

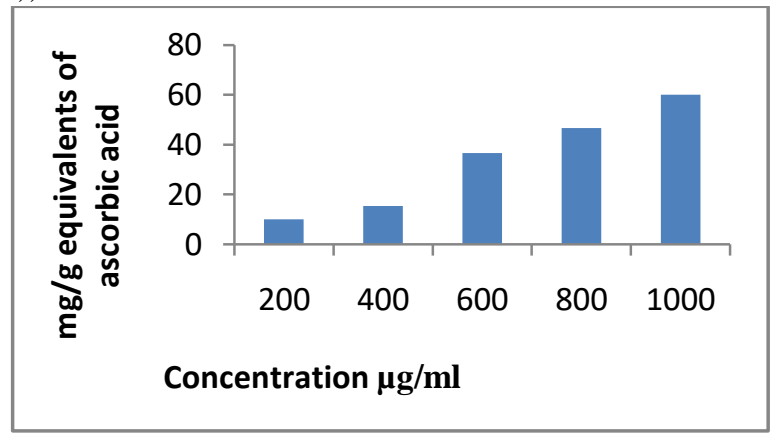

Figure :7 Total antioxidant activity of Annonamuricata

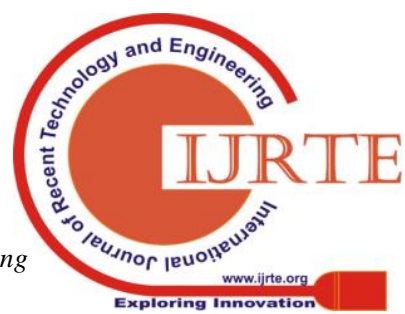


cancer prevention marketers are complement, supply a few insurance in competition to lethal facet-results and fends off illness within the body. the unconventional rummaging houses of restorative plant leaves is identified with the whole phenolic content cloth. The phosphomolybdenummethod of mobile reinforcement restriction test depending on the lower of $\mathrm{Mo}(\mathrm{VI})$ to $\mathrm{Mo}(\mathrm{V})$ via the most cancers prevention agent compound and development of a inexperienced phosphate Mo (V) complicated . From the absorbance esteem, the capability of mobile reinforcement became resolved. The effects indicated first-rate functionality of most cancers prevention agent wearing sports of silver nanoparticles.

\section{CONCLUSION}

- Silver and Copper nanoparticles have been efficiently mixed the usage of eco-accommodating, speedy, simple and ease method. included nanoparticles have been taken into consideration using visible notion, XRD, FTIR, AFM, TEM, UV-Vis and SEM techniques

- Triangular states of various sizes have been observed in the geography of AFM picture of silver and copper nanoparticles. a few lengthened oval molded debris had been available inside the geology.

- SEM snap shots of the silver nanoparticles appeared likeagglomerated circles with ugly surfaces and states of the Copper nanoparticles regarded like nanostrips with unpredictable surfaces. TEM picture likewise established that the added nano debris had been in shapes like triangle, bar and shell.The Ag nano particles have been seen spherical in form as a play around with a smooth ground morphology. The width of the nano particles changed into visible as roughly $50 \mathrm{~nm}$.

- FTIR spectra showed the nearness of phytochemicalswhich is probably in charge of the union of nanoparticles fromAnnonamuricata remove.

- The silver nanoparticles blanketed from catalyst Annonamuricata leaf pay interest proven the greater outstanding capability of cellular reinforcement carrying events .finally we presume that the leaf pay attention of Annonamuricata can be utilized by particular nourishment and pharmaceutical organizations.

\section{REFERENCES}

1. Murphy, C. J., San, T. ok.,Gole, A. M., et al., "Anisotropic metallic Nanoparticles: Synthesis, meeting, and Optical programs", magazine of bodily Chemistry B, v. 109, n. 29 , pp.13857-13870, Jun. 2005.

2. Narayanan R and El-Sayed A.M (2005) Carbon-upheld spherical palladium nanoparticles as functionality recyclable impetuses for the Suzuki response .journal of Catalysis. 234: 348.

3. Prabhuram J, Wang X, Hui LC and Hsing MI (2003) Synthesis and Characterization of Surfactant-Stabilized Pt/C Nanocatalysts for gas mobile programs. J. Phys. Chem. B. 107:11057.

4. Chandranplaystation ,Chaudhary M, Pasrichaa R, Ahmad An and Sastry M (2006) Synthesis of gold nanotriangles and silver nanoparticles the usage of Aloe vera plant separate. BiotechnolProg 22: 577.

5. Shankar S, Rai An, Ahmad An and Sastry M (2004) speedy union of $\mathrm{Au}, \mathrm{Ag}$, andbimetallic $\mathrm{Au}$ middle $\mathrm{Ag}$ shell nanoparticles making use of Neem (Azadirachtaindica) leaf juices. J. Colloid Interface.
Sci275: 496.

6. Bar H, Bhui k D, Sahoo PG, Sarkar P, Pyne S and Misra A (2009) green union of silver nanoparticles the use of seed pay attention of Jatrophacurcas. Colloids Surf. A: Physicochem. Eng. Viewpoints 348: 212.

7. Parker FS, Frost DC, Telling M, Albers P, Lopez M and Seitz k (2006) Characterisation of the adsorption locations of hydrogen on $\mathrm{Pt} / \mathrm{C}$ energy device impetuses. Catalysis nowadays 114: 418.

8. Prabhuram J, Wang X, Hui LC and Hsing MI (2003) Synthesis and Characterization of Surfactant-Stabilized Pt/C Nanocatalysts for gas cellular programs. J. Phys. Chem. B. 107:11057.

9. Liu Z, Gan ML, Hong L, Chen W and Lee YJ (2005) Carbon-upheld Pt nanoparticles as impetuses for proton exchange film electricity gadgets. J. power Sources 13:seventy 3 .

10. Saxena, A., Tripathi, R. M., and Singh, R. P. (2010). organic Synthesis of silver nanoparticles through the usage of Onion (Allium cepa) separate and their antibacterial interest.Digest. J. Nanomater. Biostruct, 5, 427-432.

11. MahmoodrezaBehravan .Et.Al., Facile inexperienced combination of silver nanoparticles making use of Berberis vulgaris leaf and root fluid pay interest and its antibacterial motion, global journal of biological Macromolecules 124 (2019) 148-154.

12. Mohammed Rafi Shaik .Et.Al., Plant-Extract-Assisted green Synthesis of Silver Nanoparticles the use of Origanumvulgare L. listen and Their Microbicidal sports Sustainability 2018, 10, 913.

13. Sah A, Juyal V, Melkani AB (2011) Antimicrobial movement of six specific portions of the plant Citrus medica Linn. Pharmacogn J three:eighty-eighty three.

14. Usman MS, Zowalaty MEE, Shameli k, Zainuddin N, Salama M, Ibrahim NA (2013) Synthesis, portrayal, and antimicrobial houses of copper nanoparticles. Int $\mathrm{J}$ Nanomed eight:4467-4479.

15. Afrah E. Mohammed, Alaa Al-Qahtani, and KawtherAabed , "Antibacterial and Cytotoxic capability of Biosynthesized Silver Nanoparticles through manner of some Plant Extracts" Nanomaterials (basel) 2018, June; $8(6) ; 382$

16. Hemanth NKS, ok.G.,Karthik L, Bhaskara RKV., "Extracellular biosynthesis of silver nanoparticles the usage of the filamentous increase Penicillium sp.". Curve ApplSci Res, 2010. 2(6): p. 161-167 